\title{
A Network Selection Strategy Based on Joint Optimization of User Satisfaction and Transmission Efficiency in Internet of Vehicle
}

\author{
Xinyi Liu, ${ }^{1,2}$ Jilong Pang, ${ }^{1,2}$ Wei Wang $\mathbb{D}^{1,2}$ Yun Meng, ${ }^{1,2}$ and Jun Hou ${ }^{1,2}$ \\ ${ }^{1}$ School of Information Engineering, Chang'an University, Xi'an 710064, Shaanxi, China \\ ${ }^{2}$ The Joint Laboratory for Internet of Vehicles, Ministry of Education, China Mobile Communications Corporation, \\ Chang'an University, Xi'an 710064, Shaanxi, China
}

Correspondence should be addressed to Wei Wang; wei.wang@chd.edu.cn

Received 15 July 2020; Revised 8 October 2020; Accepted 30 October 2020; Published 21 November 2020

Academic Editor: Haipeng Peng

Copyright (C) 2020 Xinyi Liu et al. This is an open access article distributed under the Creative Commons Attribution License, which permits unrestricted use, distribution, and reproduction in any medium, provided the original work is properly cited.

\begin{abstract}
Network selection in the Internet of Vehicles has become a popular topic of research. Unlike existing algorithms for heterogeneous network environments that rarely consider user satisfaction, in this paper, we propose a network selection strategy that takes into account both user satisfaction and transmission efficiency. We employ the effective capacity concept, which describes the maximum throughput a system can achieve under a specific statistical Quality-of-Service (QoS) delay violation probability constraint. This strategy first analyzes the influence of different utility function weight coefficients, transmission power, and time delay on each network utility satisfaction function. It is evident that the weight coefficient is proportional to the value of the utility function. Within a constrained transmission power range, the rate of increase of the function gradually slows down until it approaches a fixed value. When the delay factor value is larger, the function value is smaller, which indicates that the pursuit of lower delay will sacrifice other network performance aspects. In order to determine the maximum value of each network utility satisfaction function, a convex optimization theory is introduced for the joint optimization of user satisfaction and transmission efficiency. Finally, simulation experiments carried out under three representative network environments show that the proposed strategy is efficient and reliable.
\end{abstract}

\section{Introduction}

In recent years, Internet of Vehicle (IoV) users have developed an increasingly strong demand for high-quality access to ubiquitous broadband wireless network services [1]. This demand has created a drive for the rapid development of wireless communication technology in the IoV applications. Emerging connected vehicle and autonomous vehicle technologies can improve operational efficiency [2], mobility, and traffic safety in the entire network through Vehicle to Vehicle and Vehicle to Infrastructure Communications based on dedicated short range communications (DSRCs) [3, 4].

The Mobile communication networks have evolved from the global system for mobile communications to the universal mobile telecommunications system, followed by longterm evolution and wireless local area network (WLAN) up to the fifth generation wireless systems (5G) today [5]. At the same time, multisource heterogeneous wireless networks (MHWNs) have emerged in order to meet the needs of various services. A schematic diagram of a typical MHWN is shown in Figure 1.

In MHWNs, a variety of networks such as WLAN, cellular networks, and other types can be simultaneously selected by the user terminal. It is very critical for the users to choose an access network among these networks [6]. In MHWNs, due to the difference in the transmission quality of wireless network links, the overlap of wireless network signals, and the diversity of mobile terminal equipment, a reasonable and efficient network selection algorithm is required to formulate the IoV users' access network selection. So far, researchers in this field have conducted significant research on access selection algorithms and proposed many algorithms. In general, they can be roughly divided into 
network selection algorithms based on load balancing, received signal strength, and quality-of-service (QoS) [7]. Depending on the mathematical methods adopted, these algorithms can be further divided into those based on utility functions, neural networks, game theory, multiattribute decision making, etc. [8, 9].

Yang et al. proposed a wireless network selection scheme based on parallel transmission capability [10]. A network selection algorithm using mainly fuzzy logic and multiattribute decision-making was proposed in [11]. Singha and Singh proposed a distributed reinforcement learning strategy for selecting WLAN and WiMAX networks [12]. In [13], Pridar and Hududillah used a variety of criteria to formulate a network selection algorithm, namely, the simple weighted algorithm and the random access selection algorithm. They showed that these two algorithms provided a better network selection capability than other approaches. A network selection scheme that could select an optimal network from multisource fusion networks based on Dempster/Shafer evidence theory was proposed in [14]. Chinnappan and Balasubramanian used an analytic hierarchy process and a fuzzy logic controller for vertical switching. They proposed a network selection algorithm that considered the terminal moving speed, network cost, and load [15]. In previous studies, researchers studied resource allocation and network selection in multisource heterogeneous networks and proposed a joint resource allocation strategy using a delay factor and a prediction mechanism [16].

However, the existing methods rarely consider user satisfaction, which is an important indicator to measure service performance. It can reflect network performance and reduce the wastage of resources such as bandwidth and power. Therefore, when selecting a network, the aforementioned methods are likely to result in low QoS for the IoV users and wastage of communication resources.

In view of the above shortcomings, we propose a network selection strategy in this paper that takes into account user satisfaction and transmission efficiency. According to our simulation results, the proposed strategy can reduce the wastage of communication resources and make the network selection more efficient while guaranteeing user QoS. The main contributions of this paper are as follows:

(1) Unlike existing work, we introduce the concept of effective capacity and consider communication delay. In the effective capacity, the delay factor $\theta$ is introduced that can reflect the delay requirement of the system. To be specific, a larger $\theta$ corresponds to a faster decay rate, which implies that the system can provide a more stringent QoS requirement. On the contrary, a smaller value of $\theta$ indicates that the system has a flexible requirement for delay.

(2) To reduce the wastage of communication resources, we introduce the concept of utility function. This function can accurately reflect the relationship between the user demand and access resources, and the overall form of its expression is S-shaped.
(3) We comprehensively consider user satisfaction and transmission efficiency, as well as the delay problem by using the concept of effective capacity (EC). Experimental simulations show that the proposed network selection strategy can effectively meet the needs of different users under various network environments.

The rest of this paper is organized as follows. In Section 2, we introduce the system model and the concept of EC. We further mathematically formulate the satisfaction utility function to prove the feasibility of this feature. The formulation of the EC maximization problem under statistical delay-QoS constraint is provided in Section 3. In Section 4, we present simulations in different network environments to verify the validity and superiority of the proposed method. We summarize our findings and present future prospects in Section 5 .

\section{System Model}

Various indicators should be considered depending on the different business needs for sustainable development of communication technology and the increasing diversity of user needs. First, we need to consider not only the transmission rate but also the performance of the service it provides. As an important index for measuring service performance, user satisfaction can not only reflect the current network performance but also avoid the wastage of resources. In this paper, we propose a utility function for effectively evaluating the decision factors of network selection. As mentioned in [17], different decision factors are mapped to the corresponding utility indicators. Different IoV users require different amounts of access resources. During the early stages, users require a large amount of access resources to meet their needs. However, as the access resources increase, the user demand decreases until there is a surplus of access resources.

Second, the problem of transmission delay is very important in the IoV, as the IoV communication must first and foremost meet the delay requirements. The delay is a threshold; if the delay provided by the network is too large and exceeds the maximum delay tolerated by the user's business, it will be unacceptable even if it can provide high transmission efficiency.

Different businesses have different delay requirements. Non-real-time services such as data transfer do not have low latency requirements but require high throughput to ensure the data transfer rate. Real-time services such as video and voice communications require low latency to ensure smooth communication. In vehicular networks, vehicle control information needs to be transmitted through the network with extremely low delay and high reliability [18]. In addition, there are certain services such as web browsing, which lie between real-time and non-real-time services and require more considerations to be given to the QoS. For some networks, the extreme minimization of delay occurs at the expense of other performance aspects. According to the business characteristics, users generally choose the network 
that provides the highest efficiency and satisfaction as long as certain delay requirements are met.

The reason for choosing between multiple networks is the different performance of the services provided by each network. The service providers usually use QoS parameters to measure the service performance. In contrast, users' perception of service performance tends to be more subjective and non-technical. They expect to be served within a reasonable response time, regardless of the actual values of the technical network parameters. Therefore, in this paper, we consider user satisfaction and transmission efficiency comprehensively. We further introduce the concept of user experience satisfaction function of $\mathrm{IoV}$, which is used to characterize the demand relationship between the users and access resources, such as bandwidth and power. When the users access the network, the user experiencesatisfaction function can be expressed as follows:

$$
y(x)=\frac{\ln (1+\beta x)}{\ln (1+\beta)}, \quad \beta \geq 0, x \geq 0,
$$

where $\beta$ represents the weight of the utility function in logarithmic form. Different weights are applied to change the shape of the utility function according to user needs and network selection parameters. The current value of the access resource is represented by $x$. The satisfaction function can appropriately reflect the relationship between the access resources and the user experience function of the IoV.

The relationship between the user experience function and access resources is not linear. Initially, the access resources will be scarce, and the IoV terminals will urgently need resources to meet the current business needs. The function value is small due to the lack of access resources. With the increase of access resources, the experience satisfaction function value increases sharply. With a further increase, the function's rate of increase will be reduced. At this point, the value of the function is close to its maximum value, indicating that the access resources can meet the current business needs of the IoV terminal. A further increase in access resources will only cause minimal changes to the experience function, indicating that the terminal demand can be met at this time and the access resources are in surplus.

To sum up, as far as the terminals are concerned, the access resources do not follow a "more is better" rationale. Instead, they should be constrained to a reasonable amount, which can not only meet the needs of the IoV terminals but also avoid resource surplus and wastage. According to the utility theory, the utility function must simultaneously satisfy three conditions in order to achieve the maximum benefit. These conditions are quadratic differentiability, monotony, and concavity.

Theorem 1. The experience function shown in equation (1) is monotone, quadratically differentiable, and convex and can be used as a utility function model in this paper.

Proof. See Appendix A.
Thus, according to the above discussion, we define the user experience satisfaction function of IoV as follows:

$$
u(p)=\frac{\ln (1+\beta p)}{\ln (1+\beta)}, \quad \beta \geq 0, p \geq 0,
$$

where $p$ is the transmission power of the network.

Transmission delay is a key factor in IoV transmission. If the delay provided by the access network is larger than the maximum delay tolerated by the user, the user will consider that it is not possible to access the network. On the other hand, if the delay provided by the access network is less than or equal to the maximum delay tolerated by the user, the user may choose this network access and seek the optimal network among the accessible networks.

In this paper, the concept of EC is introduced in order to represent the transmission delay. Based on the large deviation principle, in [19], it is showed that, under sufficient conditions, the queue length process $Q(t)$ converged in distribution to a random variable $Q(\infty)$ such that

$$
-\lim _{Q_{\mathrm{th}} \longrightarrow \infty} \frac{\log \left(\operatorname{Pr}\left\{Q(\infty)>Q_{\mathrm{th}}\right\}\right)}{Q_{\mathrm{th}}}=\theta,
$$

where $Q_{\text {th }}$ is the queue length bound and the parameter $\theta>0$ is a real number. The parameter $\theta$, which is called the QoS exponent, indicates the exponential decay rate of the delaybound QoS violation probabilities. A larger $\theta$ corresponds to a faster decay rate, which implies that the system can provide a more stringent QoS requirement. A smaller $\theta$ leads to a slower decay rate, which implies a relaxed QoS requirement.

The development of wireless networks makes it possible meet the diversified business needs of the IoV users through multinetwork fusion technology. Suppose an IoV user with certain business needs is in an area simultaneously covered by $5 \mathrm{G}, \mathrm{WLAN}$, and DSRC networks. At this time, the IoV user will consider the performance of each network comprehensively, including factors such as time delay and satisfaction, and choose the most appropriate access network. We define the instantaneous transmission rate as

$$
r=T B \log _{2}\left(1+\frac{p g}{N_{0} B}\right),
$$

where $B$ is the system bandwidth and $p$ and $g$ are the transmission power and channel power gain of the user, respectively, while $N_{0}$ is the single-sided noise power spectral density (PSD) [20].

Then, the EC of the network can be expressed as follows:

$$
\begin{aligned}
C_{e}(p) & =-\frac{1}{\theta} \ln E\left[e^{-\theta T B \log _{2}(1+\gamma)}\right] \\
& =-\frac{1}{\theta} \ln I
\end{aligned}
$$

where $\gamma$ is the signal-to-noise ratio (SNR) of the user, while the expectation I will be developed later for NLOS Rayleigh fading channels, yielding equation (8).

In summary, we consider the network's transmission efficiency and user satisfaction, and after normalization, we 
obtain the utility satisfaction function over the Rayleigh fading channel (RFC) as

$$
U_{e, \mathrm{RFC}}(p)=\alpha \frac{u(p)-u_{\min }(p)}{u_{\max }(p)-u_{\min }(p)}+(1-\alpha) \frac{C_{e}(p)}{C_{e}^{\max }(p)},
$$

where $C_{e}(p) / C_{e}^{\max }(p)$ is the transmission efficiency and $\alpha$ is the weight coefficient of the utility function, whose value ranges between $0 \sim 1$. The value is selected by users according to their own business needs. If the users have a high demand for transmission efficiency, a smaller $\alpha$ will be selected, which means a higher $1-\alpha$. Consequently, the proportion of transmission efficiency will increase in the utility function. If the user's demand for transmission power is relatively high, a larger $\alpha$ will be selected and the corresponding lower $1-\alpha$ will increase the proportion of transmitting power in the utility function.

In the network selection algorithm proposed in this paper, under the premise of meeting certain delay requirements, the maximum value of the corresponding utility function of the three networks, i.e., 5G, WLAN, and DSRC, is obtained within a limited power range. Then, the user terminals select the network with the maximum utility function value as the current access network. The corresponding optimization problem to obtain the maximum utility satisfaction function value for users can be expressed as follows:

$$
\begin{gathered}
U_{e, \mathrm{RFC}}^{\mathrm{opt}}(p)=\max _{p} U_{e, \mathrm{RFC}}(p) \\
\text { s.t. } \quad \text { c.1: } C_{e}(p) \geq C_{e}^{\mathrm{min}} \\
\\
\quad \text { c.2: } p \leq p_{\max }, p \geq 0,
\end{gathered}
$$

where $C_{e}^{\min }$ represents the minimum EC under the statistical time delay QoS constraint that the user must achieve and $p_{\max }$ is the maximum transmission power of the base station (BS).

It is difficult to directly solve (7), because it is not a typical concave problem. Therefore, it is transformed as follows.

Among them,

$$
\begin{aligned}
I & =E\left[e^{-\theta T B \log _{2}(1+\gamma)}\right] \\
& =\int_{0}^{\infty} e^{-\theta T B \log _{2}\left(1+p g / N_{0} B\right)} \cdot f(g) \mathrm{d} g \\
& =\int_{0}^{\infty}\left(1+\frac{p g}{N_{0} B}\right)^{-\theta B T / \ln 2} \sigma e^{\sigma g} \mathrm{~d} g .
\end{aligned}
$$

Substituting

$$
\begin{aligned}
& t=1+g D, \\
& D=\frac{p}{N_{0} B}, \\
& A=\frac{\theta T B}{\ln 2},
\end{aligned}
$$

into (8) and assuming $\sigma=1$, we obtain

$$
\begin{aligned}
I & =\int_{0}^{\infty} e^{-g}\left(1+g_{k} D\right)^{-A} \mathrm{~d} g \\
& =\frac{e^{1 / D}}{D} \int_{1}^{\infty} e^{-(t / D)} t^{-A} \mathrm{~d} t=\frac{e^{1 / D}}{D} E_{A}\left[\frac{1}{D}\right],
\end{aligned}
$$

where $A, D>0$, and $E_{n}[x]$ is the exponential integral function. Using (5) and (10), the EC of the user for transmission over the RFC is calculated as

$$
C_{e, \mathrm{RFC}}=-\frac{1}{\theta} \ln \left(\frac{N_{0} B}{p} e^{N_{0} B / p} E_{A}\left[\frac{N_{0} B}{p}\right]\right) .
$$

Theorem 2. For Rayleigh fading channels, (11) is a concave function of $p$.

Proof. See Appendix B.

In summary, the optimal value of the utility satisfaction function can be obtained by solving (7) for any access network.

\section{Proposed Network Selection Strategy}

In this paper, under the premise of meeting certain delay requirements, we propose a network selection strategy that considers both user satisfaction and transmission efficiency. The maximum value of the utility function is obtained under different network environments. Therefore, network selection can be expressed as the following optimization problem:

$$
\begin{aligned}
U_{e, R F C}^{\mathrm{opt}} & (p)=\max _{p} U_{e, \operatorname{RFC}}(p) \\
\text { s.t. } & \text { c. } 1: C_{e}(p) \geq C_{e}^{\min } \\
& \text { c. } 2: p \leq p_{\max }, p \geq 0 .
\end{aligned}
$$

Based on Theorem 2, we can conclude that the EC maximization problem (12) is concave. Hence, it can be solved efficiently and accurately using convex optimization techniques. Therefore, we use the Lagrange multiplier method to solve the optimization problem of equation (12), as shown in (15).

In this context, the following relation holds:

$$
\underset{p}{\arg \max } U_{e, \mathrm{RFC}}(p) \equiv \arg \min _{v, \lambda} \sup _{p} L(p, v, \lambda),
$$

where the Lagrangian dual function is given by $\sup L$, i.e., the supremum of the Lagrangian. The relationship formulated in (13) is further developed in (16).

Since (16) is in a standard concave form, we can use the Karush-Kuhn-Tucker (KKT) conditions to find the optimal solution. Therefore, for given values of the Lagrange multipliers $\lambda$ and $\nu$, the optimal power allocated to the user is obtained by solving the following relation:

$$
\left.\frac{\partial L(p, v, \lambda)}{\partial p}\right|_{p=p^{*}}=0
$$

which is equivalent to finding the specific $p^{*}$ satisfying (17), which can be solved numerically using Newton's method. 
Furthermore, the dual variables may be updated using the subgradient method, as shown in (18) and (19), where $i$ is the iteration index, $\alpha_{\lambda}$ and $\alpha_{\nu}$ are the appropriate step sizes, and the operator $[\cdot]^{+}$represents $\max (0, \cdot)$.

To summarize, the specific selection strategy steps of MHWNs considering both transmission efficiency and user satisfaction are as follows:

(1) Initialization: determine the utility function weight coefficient $\alpha$, the weight value of the utility function in logarithmic form $\beta$, and the transmission power's initial value $p_{0}$ of the network, based on the user experience satisfaction function, and set the allowable error as $c$.

(2) The EC equation $C_{e}(p)=-(1 / \theta) \ln E\left[e^{-\theta T B \log _{2}(1+\gamma)}\right]$ is used based on the parameter values obtained in (1) to calculate the user's maximum transmission rate $C_{e}^{\max }$ for the different networks.

(3) Calculate $p^{i+1}$ using Newton's iterative method, where $i$ is the iteration number.

(4) Determine if $\left|p^{i+1}-p^{i}\right|<c$, and return the theoretical value of $p^{*}=p^{i+1}$. Subsequently, select the network with the maximum utility satisfaction function value as the current user access network.

(5) If $\left|p^{i+1}-p^{i}\right|>c$, then $i=i+1$ and the processing returns to step (3). The iterative algorithm continues until a power value that satisfies $\left|p^{i+1}-p^{i}\right|>c$ is obtained:

$$
\begin{aligned}
L(p, v, \lambda)= & \left((1-\alpha) \frac{-1 / \theta \ln \left(N_{0} B / p \cdot e^{N_{0} B / p} E_{A}\left[N_{0} B / p\right]\right)}{C_{e}^{\max }}+\alpha \frac{u(p)-u_{\min }(p)}{u_{\max }(p)-u_{\min }(p)}\right) \\
& +\nu\left(-\frac{1}{\theta} \ln \left(\frac{N_{0} B}{p} e^{N_{0} B / p} E_{A}\left[\frac{N_{0} B}{p}\right]\right)-C_{e, R F C}^{\min }\right)+\lambda\left(p_{\max }-p\right), \\
\sup _{p} L(p, v, \lambda)= & \arg \max _{p}\left((1-\alpha) \frac{-1 / \theta \ln \left(N_{0} B / p e^{N_{0} B / p} E_{A}\left[N_{0} B / p\right]\right)}{C_{e}^{\max }}+\alpha \frac{u(p)-u_{\min }(p)}{u_{\max }(p)-u_{\min }(p)}\right) \\
& +\nu\left(-\frac{1}{\theta} \ln \left(\frac{N_{0} B}{p} e^{N_{0} B / p} E_{A}\left[\frac{N_{0} B}{p}\right]\right)-C_{e, \mathrm{RFC}}^{\min }\right)+\lambda\left(p_{\max }-p\right) \\
= & \arg \max _{p}\left(-\frac{\nu C_{e}^{\max }+1-\alpha}{\theta C_{e}^{\max }} \ln \left(\frac{N_{0} B}{p} e^{N_{0} B / p} E_{A}\left[\frac{N_{0} B}{p}\right]\right)+\alpha \frac{u(p)}{u_{\max }(p)-u_{\min }(p)}-\lambda p\right), \\
& -\frac{1}{p^{2}}\left[\frac{N_{0} B\left(\nu C_{e}^{\max }+1-\alpha\right)}{\theta C_{e}^{\max }}+\frac{p\left(\nu C_{e}^{\max }+1-\alpha\right)}{\theta C_{e}^{\max }}+\lambda p^{2}-\left(\frac{N_{0} B\left(\nu C_{e}^{\max }+1-\alpha\right)}{\theta C_{e}^{\max }} \frac{E_{A-1}\left[N_{0} B / p\right]}{E_{A}\left[N_{0} B / p\right]}\right)\right] \\
& -\left(\alpha \frac{\beta /[\ln (1+\beta)](1+\beta p)}{u_{\max }(p)-u_{\min }(p)}\right)=0 .
\end{aligned}
$$

\section{Simulation Results and Analysis}

4.1. Simulation Environment. We used Matlab 2016 for performing the simulation experiments. Considering an urban environment, network selection was carried out between three networks, namely, 5G, WLAN, and DSRC.

4.2. Simulation Results. Figure 2 shows the variation trend of experience satisfaction function for different access resources.
As can be seen from the figure, the function is monotonous and concave. In general, as the access resource value increases, the value of the utility satisfaction function initially increases sharply. This sharp increase occurs because the IoV terminal is in urgent need of access resources to meet the current business needs. However, due to the lack of access resources, the value of the satisfaction experience function remains small. The rate of increase of the function becomes very small as the access resources increase. At this point, the change of the access resource value will only marginally increase the function. A comparison shows that the function shape changes as the value of $\beta$ changes. The 


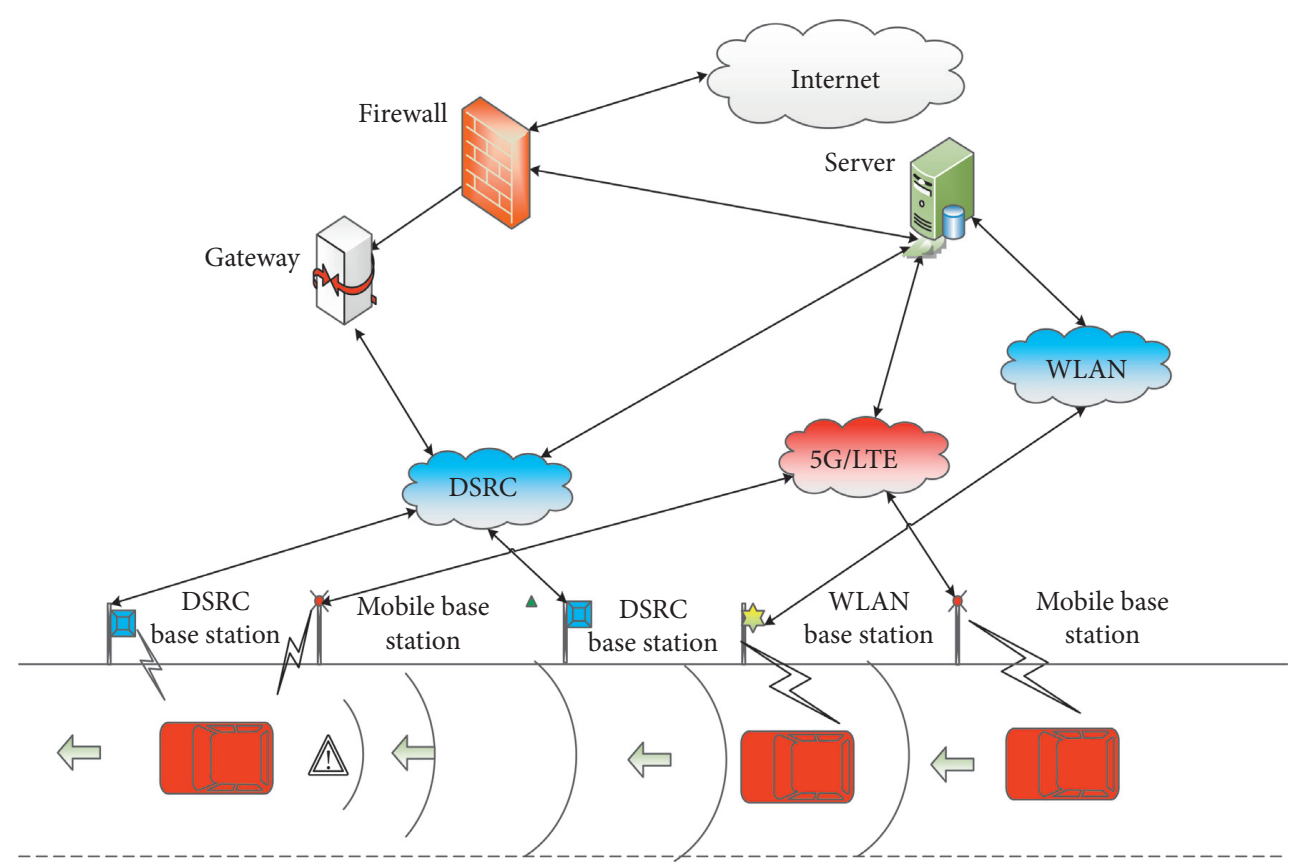

FIGURE 1: Architecture of multisource heterogeneous vehicle network.

IoV terminal can select different values according to its business requirements.

$$
\begin{aligned}
\lambda(i+1) & =\left[\lambda(i)-\alpha_{\lambda}(i)\left(\frac{\partial L(p, \nu, \lambda)}{\partial \lambda}\right)\right]^{+} \\
& =\left[\lambda(i)-\alpha_{\lambda}(i)\left(p_{\max }-p\right)\right]^{+} \\
\nu(i+1) & =\left[\nu(i)-\alpha_{\nu}(i)\left(\frac{\partial L(p, \nu, \lambda)}{\partial \nu}\right)\right]^{+} \\
& =\left[\nu(i)-\alpha_{\nu}(i)\left(-\frac{1}{\theta} \ln \left(\frac{N_{0} B}{p} e^{N_{0} B / p} E_{A}\left[\frac{N_{0} B}{p}\right]\right)-C_{e, \operatorname{RFC}}^{\min }\right)\right]^{+} .
\end{aligned}
$$

Figure 3 shows the relationship between the user utility satisfaction function value and transmission power for different values of $\alpha$ and $\theta$.

Generally speaking, the value of the utility function increases as the transmission power increases and finally approaches a maximum value. First, we examine the influence of satisfaction and transmission efficiency on network performance under the same business demands. A small value of $\alpha$ is required when the terminal has a high transmission efficiency requirement. It can be seen that as $\alpha$ decreases, $1-\alpha$ increases. The greater the value of $1-\alpha$, the smaller the value of the satisfaction utility function obtained by the IoV user.

Next, we examine how the value of the utility function changes for certain proportions of the two parts (namely, user satisfaction and transmission efficiency) and different delay factors. Let $\alpha=0.5$ and $\theta=0.5$. It can be seen that, when the value of $\theta$ is large, the strictness of the delay requirement is higher, and lower delay values will be at the expense of other network performance aspects. When $\alpha=$ 0.5 and $\theta=0.1$, the satisfaction function value is higher.

Figure 3 examines the effect of the delay factor $\theta$ and weight coefficient $\alpha$ on the utility function under the same network environment. Figure 4 analyzes how the value of the utility function changes under different network environments for the same service requirements. Therefore, letting $\alpha=0.5$ and $\theta=0.1$, we see that the value of the utility function increases as the transmission power increases. The function value obtained in the WLAN environment is the largest, so it is the selected network for the current user's access. 


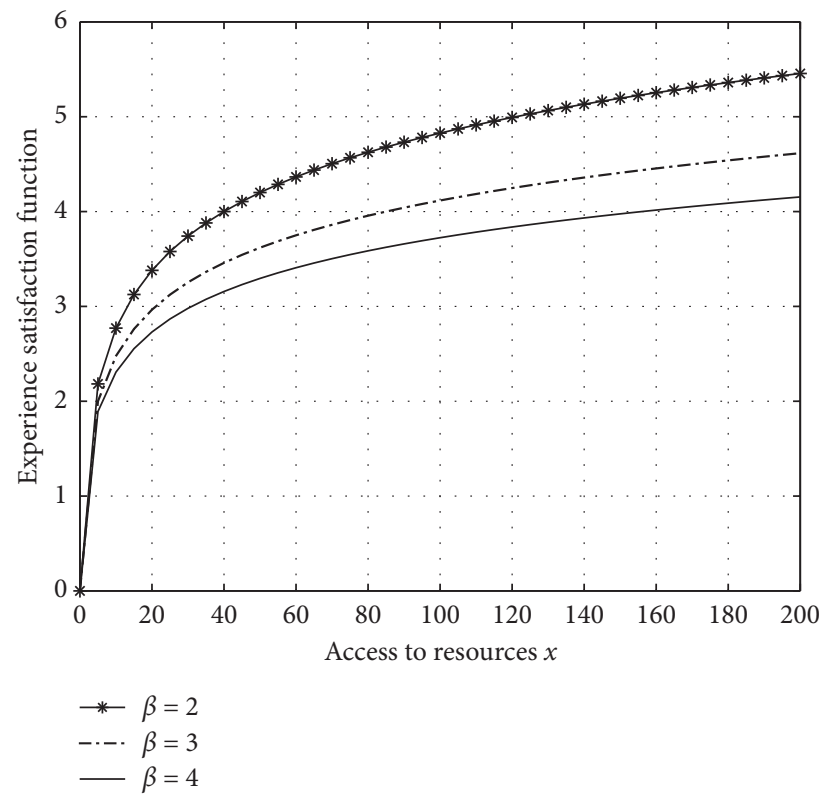

Figure 2: Variation trend of experience satisfaction function for different access resources.

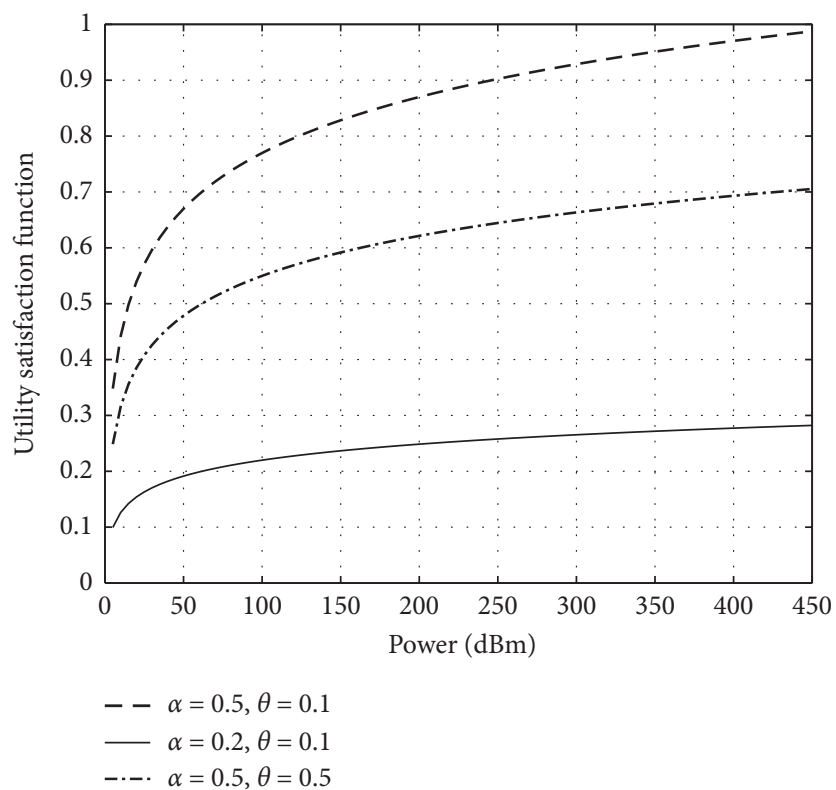

FIGURE 3: Relationship between user utility satisfaction function and transmission power for different values of $\alpha$ and $\theta$.

Figure 5 is a comparison of the utility satisfaction function for different network selection strategies.

In this case, a single variable value remains constant. Letting $\theta=0.1$ and $\alpha=0.5$, we compare the network selection strategy proposed in this paper with the network selection strategies based on transmission power, transmission efficiency, and throughput. As evident from the figure, within a certain power range, e.g., $0-250 \mathrm{dBm}$, the utility satisfaction function value obtained by the proposed network selection strategy is the largest, followed by that obtained using transmission power and transmission efficiency. The smallest value of the utility satisfaction function is obtained using throughput. When the power exceeds
$250 \mathrm{dBm}$, the value of the utility function obtained by the throughput-based network selection strategy will exceed that obtained by the transmission efficiency strategy. However, the value obtained by the former strategy will eventually approach a fixed value.

To sum up, the strategy proposed in this paper can give the highest utility function value. This is because it considers both transmission power and transmission efficiency and combines the two through a functional relationship, resulting in superior performance.

In summary, when the IoV users have access to different networks, a curve with the maximum satisfaction utility function value can be obtained under different conditions. The 


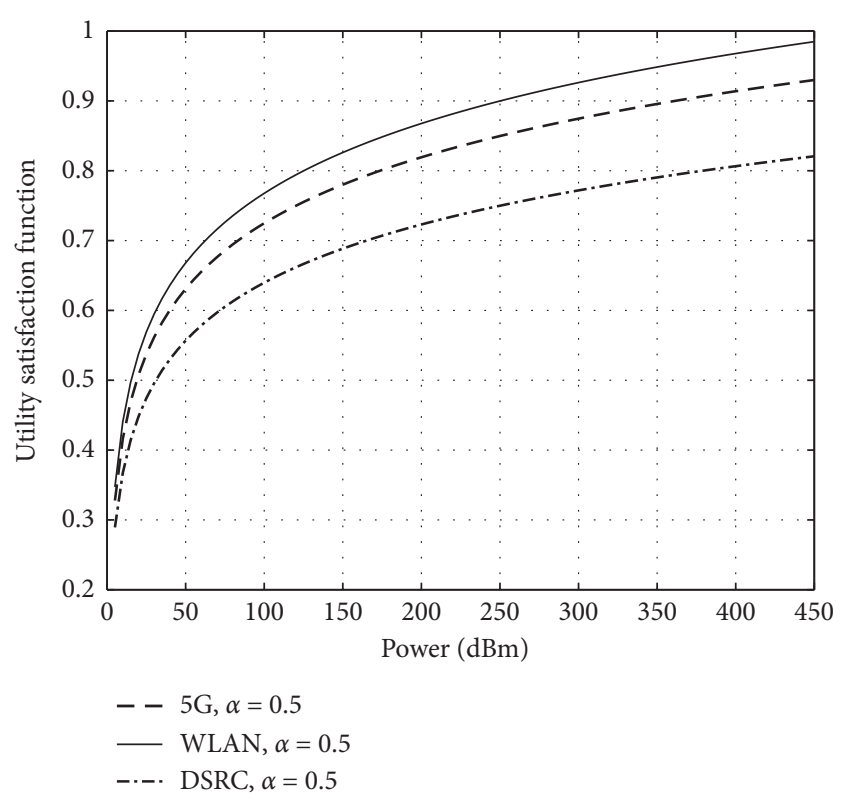

FIgURE 4: Relationship diagram of utility function values in different network environments.

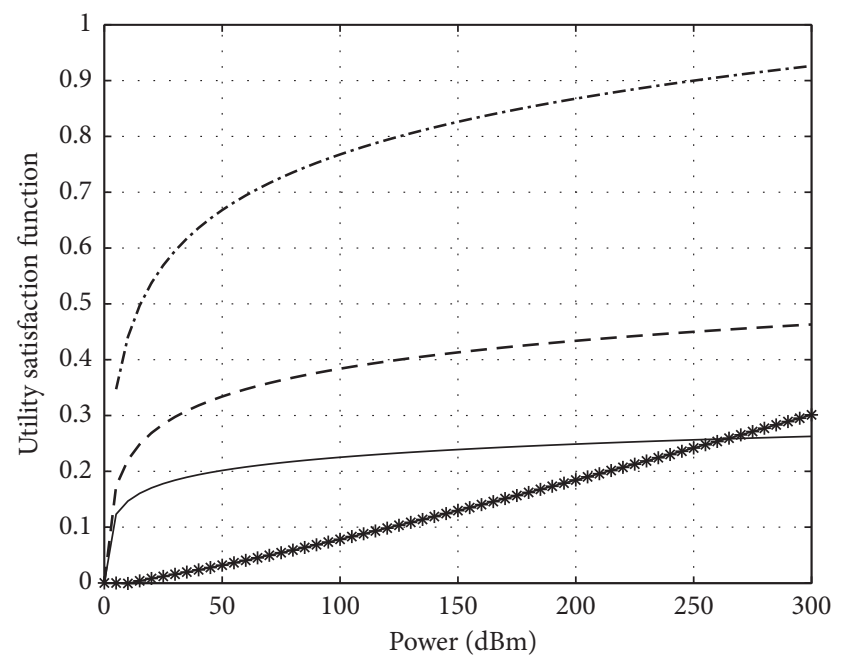

- - Network selection strategy based on transmitting power

_ Network selection strategy based on transmission efficiency

* Network selection strategy based on throughput

-.-. The proposed network selection strategy

Figure 5: Comparison of utility satisfaction function values for different network selection strategies.

network corresponding to the current maximum utility satisfaction function value is selected as the current access network of the IoV user. Therefore, the proposed strategy can effectively and reliably solve the problem of network selection.

\section{Conclusion}

Many algorithms have been proposed for solving the problem of optimal scheduling of IoV terminals in heterogeneous network environments. However, only a few algorithms consider user satisfaction. User satisfaction is an important index for measuring the service performance, and it can not only reflect the current network's performance but also avoid the wastage of resources. Therefore, in this paper, we proposed a network selection strategy under heterogeneous network environments, which considered both user satisfaction and transmission efficiency under the premise of satisfying certain delay requirements. The experimental results showed that the proposed strategy could not only ensure user satisfaction but also resulted in more efficient use of communication resources. Therefore, it solved the problem of optimal scheduling of different services to IoV terminals in heterogeneous network environments.

The proposed strategy has certain reference values for the access selection algorithm based on multiple factors. In order to solve the problem of network selection in heterogeneous network environments more effectively and efficiently, other decision factors such as energy consumption, throughput, and packet loss rate will be considered in future research.

\section{Appendix}

\section{A. Proof of Theorem 1}

Here, we use mathematical methods to prove that the satisfaction experience is a twice differentiable monotonic convex function.

For the following function,

$$
y(x)=\frac{\ln (1+\beta x)}{\ln (1+\beta)}, \quad \beta \geq 0, x \geq 0,
$$

the first derivative is

$$
y^{\prime}(x)=\frac{\beta}{[\ln (1+\beta)](1+\beta x)}>0 .
$$

Therefore, the function is monotonic.

According to equation (A.2), the second derivative is

$$
\begin{aligned}
y^{\prime \prime}(x) & =\frac{\beta}{\ln (1+\beta)} \cdot \frac{-\beta^{2}}{1+\beta x} \\
& =\frac{-\beta^{3}}{[\ln (1+\beta)](1+\beta)^{2}}<0 .
\end{aligned}
$$

Based on (A.3), we can observe that the experience satisfaction function of equation (A.1) is twice differentiable and concave. Therefore, we choose the above function as a model utility function. The simulation results are shown in Figure 2.

The proof is concluded.

\section{B. Proof of Theorem 2}

For the following function, 


$$
C_{e, R F C}=-\frac{1}{\theta} \ln \left(\frac{N_{0} B}{p} e^{N_{0} B / p} E_{A}\left[\frac{N_{0} B}{p}\right]\right),
$$

the first and second derivatives are shown in (B.2) and (B.3), respectively. Therefore, equation (B.1) is a concave function of $p$ for Rayleigh fading channels.

The proof is concluded.

$$
\begin{gathered}
C_{e, \operatorname{RFC}}(p)=-\frac{N_{0} B}{\theta p^{2}}-\frac{1}{\theta p}+\frac{N_{0} B}{\theta p^{2}} \cdot \frac{E_{A-1}\left[N_{0} B / p\right]}{E_{A}\left[N_{0} B / p\right]}, \\
C_{e, \mathrm{RFC}}^{\prime \prime}(p)=\frac{e^{-\left(2 N_{0} B / p\right)} p-e^{-\left(N_{0} B / p\right)}\left(N_{0} B+(2-A) p\right) E_{A}\left[N_{0} B / p\right]-\left(2 N_{0} B+A p\right) E_{A}\left[N_{0} B / p\right]^{2}}{\theta p^{3} E_{A}\left[N_{0} B / p\right]^{2}} \leq 0 .
\end{gathered}
$$

\section{Data Availability}

The data used to support the findings of this study are available from the corresponding author upon request.

\section{Conflicts of Interest}

The authors declare that they have no conflicts of interest.

\section{Acknowledgments}

This work was supported by the National Natural Science Foundation of China (nos. 61803041, 61871059, and 61701044), The Key R\&D Program in Shaanxi Province under No. 2020GY-011, China Postdoctoral Science Foundation (no. 2017M623089), and Xi'an Science and Technology Plan Project (no. 2019218514GXRC021CG022-GXYD21.4).

\section{References}

[1] T. Han, X. Ge, L. Wang, K. S. Kwak, Y. Han, and X. Liu, "5G converged cell-less communications in smart cities," IEEE Communications Magazine, vol. 55, no. 3, pp. 44-50, 2017.

[2] T. S. J. Darwish and K. Abu Bakar, "Fog based intelligent transportation big data analytics in the internet of vehicles environment: motivations, architecture, challenges, and critical issues," IEEE Access, vol. 6, no. 99, pp. 15679-15701, 2018.

[3] L. Xu, L. Huang, C. Cao, H. Wang, Y. Li, and T. A. Gulliver, "Outage performance of mobile V2V cooperative networks," Physical Communication, vol. 34, pp. 295-300, 2019.

[4] Y. Wang and C. Wei, "A universal trajectory planning method for automated lane-changing and overtaking maneuvers," Mathematical Problems in Engineering, vol. 2020, Article ID 1023975, 13 pages, 2020.

[5] S. Li, L. D. Xu, and S. Zhao, "5G Internet of things: a survey," Journal of Industrial Information Integration, vol. 10, pp. 1-9, 2018.

[6] C. S. Voon, K. H. Yeap, K. C. Lai et al., "A compact doublepsi-shaped dual band patch antenna for WLAN/LTE applications," Microwave \& Optical Technology Letters, vol. 60, no. 5, pp. 1271-1275, 2018.

[7] M. Liu, Y. Shao, C. Yu, and J. Yu, "A heterogeneous QoSbased cloud service selection approach using entropy weight and GRA-ELECTRE III," Mathematical Problems in Engineering, vol. 2020, Article ID 1536872, 17 pages, 2020.

[8] Y.-C. Chang, C. S. Chang, C.-S. Chang, and J.-P. Sheu, "An enhanced fast multi-radio rendezvous algorithm in heterogeneous cognitive radio networks," Institute of Electrical and Electronics Engineers Transactions on Cognitive Communications and Networking, vol. 4, no. 4, pp. 847-859, 2018.

[9] S. Fu, X.-l. Qu, H.-j. Zhou, and G.-b. Fan, "A multi-attribute decision-making model using interval-valued intuitionistic fuzzy numbers and attribute correlation," International Journal of Enterprise Information Systems, vol. 14, no. 1, pp. 21-34, 2018.

[10] B. Yang, P. S. Wei, C. E. Mcelcheran et al., "A platform for 4channel parallel transmission MRI at 3T: demonstration of reduced radiofrequency heating in a test object containing an implanted wire," Journal of Medical and Biological Engineering, vol. 39, no. 5, pp. 1-10, 2019.

[11] A. Jamshidi-Zanjani and M. Rezaei, "Landfill site selection using combination of fuzzy logic and multi-attribute decisionmaking approach," Environmental Earth Sciences, vol. 76, no. 13, pp. 448-461, 2017.

[12] N. Singha and Y. N. Singh, "Optimal capacity partitioning in homogeneous P2P network," IEEE Communications Letters, vol. 22, no. 7, pp. 1354-1357, 2018

[13] R. Pridar and T. H. Hududillah, "Risk level analysis of lightning strike with simple additive weighting method in gowa region," Jurnal Penelitian Fisika Dan Aplikasinya, vol. 8, no. 1, pp. 17-24, 2018.

[14] P. Dutta, "Health risk assessment through incorporation of dempster-shafer structure and fuzzy numbers within the same framework," New Mathematics and Natural Computation, vol. 15, no. 2, pp. 231-259, 2019.

[15] A. Chinappan and R. Balasubramanian, "Complexity-consistency trade-off in multi-attribute decision making for vertical handover in heterogeneous wireless networks," Institution of Engineering and Technology Networks, vol. 5, no. 1, pp. 13-21, 2016.

[16] L. Jiandong, J. Jian, and L. Xinyi, "A network selection policy under delay constraint and resource prediction in integrated wireless systems," Journal of Xi'an Jiaotong University, vol. 48, no. 2, pp. 74-79, 2014.

[17] D. Jiang, L. Huo, Z. Lv et al., "A joint multi-criteria utility-based network selection approach for vehicle-to-infrastructure networking," Institute of Electrical and Electronics Engineers Transactions on Intelligent Transportation Systems, vol. 99, pp. 1-15, 2018.

[18] W. Wang, Y. Wang, J. Dai, and Z. Cao, "Dynamic soft realtime scheduling with preemption threshold for streaming 
media," International Journal of Digital Multimedia Broadcasting, vol. 2019, Article ID 5284968, 7 pages, 2019.

[19] C. S. Chang, "Stability, queue length, and delay of deterministic and stochastic queueing networks," Institute of Electrical and Electronics Engineers Transactions on Automatic Control, vol. 39, no. 5, pp. 913-931, 1994.

[20] C. E. Shannon, "A mathematical theory of communication," Bell System Technical Journal, vol. 27, no. 3, pp. 379-423, 1948. 